PHYSICAL REVIEW D 92, 072009 (2015)

\title{
Local-oscillator noise coupling in balanced homodyne readout for advanced gravitational wave detectors
}

\author{
Sebastian Steinlechner, Bryan W. Barr, Angus S. Bell, Stefan L. Danilishin, Andreas Gläfke, Christian Gräf, \\ Jan-Simon Hennig, E. Alasdair Houston, Sabina H. Huttner, Sean S. Leavey, Daniela Pascucci, Borja Sorazu, \\ Andrew Spencer, Kenneth A. Strain, Jennifer Wright, and Stefan Hild \\ SUPA, School of Physics and Astronomy, The University of Glasgow, Glasgow G12 8QQ, United Kingdom
}

(Received 25 June 2015; published 20 October 2015)

\begin{abstract}
The second generation of interferometric gravitational wave detectors are quickly approaching their design sensitivity. For the first time these detectors will become limited by quantum backaction noise. Several backaction evasion techniques have been proposed to further increase the detector sensitivity. Since most proposals rely on a flexible readout of the full amplitude- and phase-quadrature space of the output light field, balanced homodyne detection is generally expected to replace the currently used DC readout. Up to now, little investigation has been undertaken into how balanced homodyne detection can be successfully transferred from its ubiquitous application in tabletop quantum optics experiments to largescale interferometers with suspended optics. Here we derive implementation requirements with respect to local-oscillator noise couplings and highlight potential issues with the example of the Glasgow Sagnac Speed Meter experiment, as well as for a future upgrade to the Advanced LIGO detectors.
\end{abstract}

DOI: 10.1103/PhysRevD.92.072009

PACS numbers: 04.80.Nn, 07.60.Ly

\section{INTRODUCTION}

A century after gravitational waves were first predicted by Albert Einstein as a result of his general theory of relativity [1], the worldwide effort to provide the first direct detection of these waves is still ongoing. The Advanced LIGO generation of interferometric gravitational wave detectors has just left its construction stages and is now being brought to their design sensitivity [2].

Contrary to previous generations, these detectors will be limited by quantum noise over their whole detection bandwidth. Above about $50 \mathrm{~Hz}$, shot noise from the photon statistics will be dominant, while at lower frequencies the sensitivity will be limited by backaction noise. In anticipation of these limitations, several technologies have been devised over the past years to specifically target and reduce quantum noise. Squeezed states of light are the most mature of these technologies and their application in gravitational wave detectors has been successfully demonstrated in GEO 600 and LIGO [3,4] and intensely studied over the past few years [5].

To target the full quantum-noise spectrum, squeezing has to be combined with filter cavities that shape the quantum correlations such that an optimal backaction suppression is achieved at each frequency [6]. Furthermore, topologies such as variational readout have been proposed where also the quadrature of the detected output light field is adjusted

Published by the American Physical Society under the terms of the Creative Commons Attribution 3.0 License. Further distribution of this work must maintain attribution to the author(s) and the published article's title, journal citation, and DOI. in a frequency-dependent way [6]. This is an example of a quantum nondemolition measurement, which theoretically allows for a backaction noisefree readout [7]. Another example for such a measurement is the speed meter configuration, where the velocity of the test masses is detected instead of their position [8]. It exploits that the momentum - and the derived quantity velocity — of a free test mass is conserved, and thus repeated measurements of the speed do not influence each other. In 2003, Y. Chen showed that the Sagnac interferometer configuration is a close approximation of a speed meter [9], and experiments are now underway to demonstrate the backaction evasion properties of Sagnac interferometers [10].

In these advanced technologies, it is generally assumed that the detected field quadrature of the output signal can be freely adjusted. Only then can the optimal balance between quantum noise and detected signal be achieved, which is essential for the goal to surpass the standard quantum limit. This is, however, not possible with the current DC readout technique $[11,12]$, which is predominantly sensitive to the signal quadrature. Balanced homodyne (BHD) detection [13] would allow for precisely choosing the readout quadrature [14], and it is a well-proven workhorse for tabletop experiments throughout quantum optics [15]. It has been shown to provide shot-noise limited sensitivity over a wide frequency band from the $\mathrm{GHz}$ range [16] down to the sub-Hz regime [17].

However, attention has only recently turned to the problem of how to implement a BHD readout in largescale interferometers [18]. Here, we investigate the noise requirements that have to be imposed on such a readout scheme. In particular, we focus on the case of significant amounts of carrier light (on the order of a $\mathrm{mW}$ ) in the 


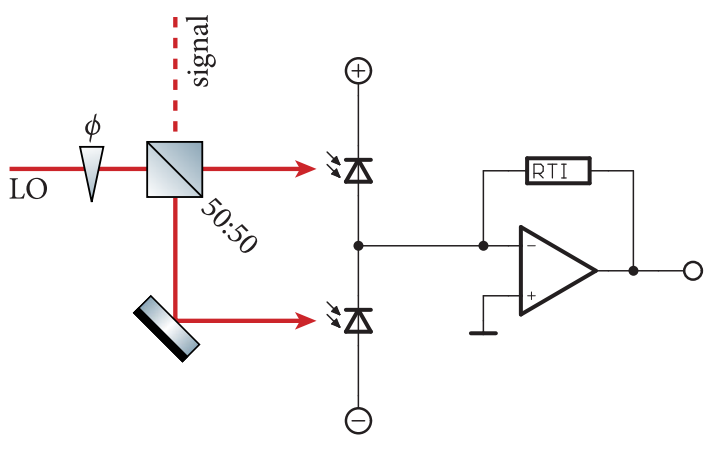

FIG. 1 (color online). Schematic of balanced homodyne detection. The signal field is overlapped on a 50:50 beam splitter with a strong LO. Both beam-splitter outputs are detected with a high-efficiency photodiode and subtracted from each other. Here, a direct photocurrent subtraction circuit is shown. The readout quadrature can be selected by adjusting the relative phase $\phi$ between signal and LO field. RTI, transimpedance-gain setting resistor.

interferometer output field. This carrier light is introduced by a contrast defect of the main interferometer (e.g., differential losses of the interferometer arms) and, as we will show below, can lead to significant requirements on local-oscillator stability.

\section{FUNDAMENTALS OF BALANCED HOMODYNE DETECTION}

For the following discussion, a simple review of the quantum-mechanical description of balanced homodyne detection is helpful. Following, e.g., [19], we define the amplitude- and phase-quadrature operators $\boldsymbol{X}_{1}=$ $\left(\boldsymbol{a}+\boldsymbol{a}^{\dagger}\right) / 2, \boldsymbol{X}_{2}=-\mathrm{i}\left(\boldsymbol{a}-\boldsymbol{a}^{\dagger}\right) / 2$, where $\boldsymbol{a}$ is the annihilation operator for a single-mode optical field. Furthermore, we set $\boldsymbol{a}=\alpha+\delta \boldsymbol{a}$, which separates the mode's classical amplitude $\alpha$ from its quantum fluctuations $\delta \boldsymbol{a}$. In a step that is commonly referred to as linearization, terms of higher than linear order in the noise are neglected, i.e., $\delta \boldsymbol{a} \delta \boldsymbol{b} \rightarrow 0$.

In balanced homodyne detection, the signal beam $\boldsymbol{b}$ is overlapped at a 50:50 beam splitter with a strong localoscillator (LO) field $\boldsymbol{a}$ as shown in Fig. 1. Without loss of generality, we can assume their coherent amplitudes $\alpha$ and $\beta$, respectively, to be real, and absorb the relative phase $\phi$ between the two fields in a phase factor $\exp (\mathrm{i} \phi)$. The intensity in the two beam-splitter output ports $\boldsymbol{c}$ and $\boldsymbol{d}$ is then given by

$$
\begin{aligned}
\boldsymbol{c}^{\dagger} \boldsymbol{c}= & \frac{1}{2}\left[\boldsymbol{a}^{\dagger} \boldsymbol{a}+\boldsymbol{a}^{\dagger} \boldsymbol{b} \mathrm{e}^{-\mathrm{i} \phi}+\boldsymbol{a} \boldsymbol{b}^{\dagger} \mathrm{e}^{\mathrm{i} \phi}+\boldsymbol{b}^{\dagger} \boldsymbol{b}\right] \\
= & \frac{1}{2}\left[\alpha^{2}+\beta^{2}+2 \alpha \beta \cos \phi+2 \alpha \delta \boldsymbol{X}_{1}^{a}+2 \beta \delta \boldsymbol{X}_{1}^{b}\right. \\
& \left.+2 \alpha \delta \boldsymbol{X}_{-\phi}^{b}+2 \beta \delta \boldsymbol{X}_{\phi}^{a}\right]
\end{aligned}
$$

and

$$
\begin{aligned}
\boldsymbol{d}^{\dagger} \boldsymbol{d}= & \frac{1}{2}\left[\alpha^{2}+\beta^{2}-2 \alpha \beta \cos \phi+2 \alpha \delta \boldsymbol{X}_{1}^{a}+2 \beta \delta \boldsymbol{X}_{1}^{b}\right. \\
& \left.-2 \alpha \delta \boldsymbol{X}_{-\phi}^{b}-2 \beta \delta \boldsymbol{X}_{\phi}^{a}\right] .
\end{aligned}
$$

Here, $\boldsymbol{X}_{\phi}=\boldsymbol{X}_{1} \cos \phi+\boldsymbol{X}_{2} \sin \phi$ is the quadrature operator for the quadrature angle $\phi$. A more detailed calculation can be found in Appendix A.

Both output ports $\boldsymbol{c}$ and $\boldsymbol{d}$ are separately detected with high-efficiency photodetectors and the resulting photocurrents are subtracted, yielding a detector output that is proportional to

$$
\boldsymbol{i}_{-}=\boldsymbol{c}^{\dagger} \boldsymbol{c}-\boldsymbol{d}^{\dagger} \boldsymbol{d}=2 \alpha \beta \cos \phi+2 \alpha \delta \boldsymbol{X}_{-\phi}^{b}+2 \beta \delta \boldsymbol{X}_{\phi}^{a}
$$

with a noise variance of

$$
\Delta^{2} \boldsymbol{i}_{-}=4 \alpha^{2} \Delta^{2} \delta \boldsymbol{X}_{-\phi}^{b}+4 \beta^{2} \Delta^{2} \delta \boldsymbol{X}_{\phi}^{a}
$$

The power in the local oscillator $P_{\mathrm{LO}}=\alpha^{2}$ is chosen to be much larger than the power in the signal field $P_{\text {sig }}=\beta^{2}$,

$$
P_{\mathrm{LO}} \gg P_{\text {sig. }}
$$

Therefore, the last term in Eq. (5) can usually be neglected. The output is then directly proportional to the signal's noise in the quadrature $\boldsymbol{X}_{-\phi}^{b}$, amplified by the coherent amplitude of the local oscillator. Tuning the relative phase $\phi$ between local oscillator and signal allows for an easily accessible adjustment of the detected quadrature.

Fulfilling the condition (6) is, however, not sufficient when the noise variance of the local-oscillator beam is much higher than the signal's noise variance. Usually, the signals that one tries to measure with balanced homodyne detection are very close to or even below the quantummechanical zero-point fluctuations, i.e., $\Delta^{2} \boldsymbol{\delta} X_{\phi}^{b} \approx \hbar \omega / 4$. At the same time, the local oscillator will be orders of magnitude away from the shot-noise limit due to technical laser noise, unless the measurement frequencies are well above the laser's relaxation oscillation, or else significant effort has been put into laser stabilization [20]. Therefore, even tiny amounts of carrier light in the signal field can amplify the local-oscillator's noise sufficiently such that it completely dominates the output of the balanced homodyne detector. In the following section we will derive requirements on the amplitude and phase stability of the local oscillator, depending on the residual power $P_{\text {sig }}$ in the signal beam. 


\section{NOISE COUPLING MECHANISMS IN BALANCED HOMODYNE DETECTION}

\section{A. Amplitude noise}

Current DC readout schemes set strong requirements to the laser amplitude noise level: to provide the localoscillator light, the interferometer output port has to operate at some DC offset, thus sending a fraction of the light from the laser directly towards the output detector.

Balanced homodyne readout also uses a local oscillator at the carrier frequency and is thus, in principle, susceptible to laser amplitude noise. However, this noise is common to both photodiodes and can thus be subtracted out by careful balancing of the photocurrents. We can see this behavior by putting an imbalance term $1-\varepsilon$ into (4); see also Appendix B for more details:

$$
\begin{aligned}
\boldsymbol{i}_{-, \varepsilon} & =\boldsymbol{c}^{\dagger} \boldsymbol{c}-(1-\varepsilon) \boldsymbol{d}^{\dagger} \boldsymbol{d} \\
& =\mathrm{const}+\varepsilon \alpha \delta \boldsymbol{X}_{1}^{a}+(2-\varepsilon) \alpha \delta \boldsymbol{X}_{-\phi}^{b} .
\end{aligned}
$$

Any difference in, e.g., the beam-splitting ratio and photodiode quantum efficiency thus adds noise from the local oscillator's amplitude quadrature $\boldsymbol{X}_{1}^{a}$. In practice, $\varepsilon$ can be made very small and common-mode suppression ratios of more than $80 \mathrm{~dB}$ have been demonstrated [21].

The situation gets worse, however, when the signal field carries significant DC power, i.e., $\beta \gg 0$. Such a situation quickly arises due to a small contrast defect of the interferometer. Here we derive a relative intensity noise (RIN) requirement for the LO beam as follows. Looking at the noise variance in the output signal (5), we see that the noise in the signal field is amplified by the LO carrier, while noise in the LO field is amplified by the signal carrier. We set $\phi=0$ as we are only concerned with noise in the amplitude quadrature for now. The output should be dominated by the noise in the signal; therefore, we require

$$
4 P_{\mathrm{LO}} \Delta^{2} \delta \boldsymbol{X}_{1}^{b}>4 P_{\mathrm{sig}} \Delta^{2} \delta \boldsymbol{X}_{1}^{a} .
$$

After dividing both sides by $P_{\mathrm{LO}} \times P_{\text {sig }}$, i.e., the product of the mean (DC) powers in both signal and LO, this takes on the form of

$$
\mathrm{RIN}_{\text {sig }}^{2}>\mathrm{RIN}_{\mathrm{LO}}^{2}
$$

where

$$
\mathrm{RIN}=\frac{\Delta P}{P}=\frac{\sqrt{P \Delta^{2} \delta \boldsymbol{X}_{1}}}{P}
$$

is the relative intensity noise of the respective beam. Finally, for signals at the quantum-noise limit, RIN $_{\text {sig }}$ is just the shot-noise limited RIN, $\mathrm{RIN}_{\mathrm{SN}, \mathrm{sig}}=\sqrt{2 \hbar \omega / P_{\text {sig }}}$, and thus results in the requirement

$$
\mathrm{RIN}_{\text {LO }}<\sqrt{\frac{2 \hbar \omega}{P_{\text {sig }}}} .
$$

Since balanced homodyne detection requires $P_{\mathrm{LO}}>P_{\text {sig }}$, this inequality is always fulfilled as long as the LO field is shot noise limited. This is usually not a problem in tabletop experiments in quantum optics where typical signals occur at sideband frequencies of several $\mathrm{MHz}$, well away from technical noise sources such as the laser's relaxation oscillation. For signals in the audio band, however, it is much more difficult to get close to a shot-noise limited RIN [20] so that the condition (12) can pose a challenging requirement.

\section{B. Local-oscillator path-length stability}

Let us now address phase noise within the localoscillator beam. In particular, since the LO and signal beams will have to travel along spatially separate paths for at least some distance, we will derive a requirement for the differential length fluctuations between these two paths.

Again, from Eq. (5) we get the requirement that

$$
P_{\text {sig }} \Delta^{2} X_{2}^{\mathrm{LO}}<P_{\mathrm{LO}} \Delta^{2} \boldsymbol{X}_{2}^{\mathrm{sig}} \approx P_{\mathrm{LO}} \frac{\hbar \omega}{4},
$$

such that the BHD output is not limited by excess noise on the LO beam. In the last step, we assumed a signal that is close to the vacuum noise, i.e., $\Delta^{2} \boldsymbol{X}_{2}^{\text {sig }} \approx \hbar \omega / 4$. If a (suspended) mirror in the LO path moves by a distance $\Delta x$, the corresponding signal in the phase quadrature is given by [22]

$$
\boldsymbol{X}_{2}^{\mathrm{LO}}=\frac{2 \omega}{c} \sqrt{P_{\mathrm{LO}}} \Delta x .
$$

Inserting this into (13) leads to

$$
P_{\mathrm{sig}} \frac{4 \omega^{2} P_{\mathrm{LO}}}{c^{2}} \Delta^{2} x<P_{\mathrm{LO}} \frac{\hbar \omega}{4}
$$

and thus

$$
\Delta^{2} x<\frac{\hbar c^{2}}{16 P_{\mathrm{sig}} \omega}
$$

which is, perhaps surprisingly, independent of $P_{\mathrm{LO}}$. Equivalently, we get the requirement for the single-sided displacement spectral density

$\tilde{x}(f)<\sqrt{\frac{\hbar c^{2}}{8 \omega P_{\text {sig }}}} \approx 8.2 \times 10^{-16} \mathrm{~m} / \sqrt{\mathrm{Hz}} \times \sqrt{\frac{1 \mathrm{~mW}}{P_{\text {sig }}}}$

assuming a carrier frequency of $\omega=2 \pi c / 1064 \mathrm{~nm}$. This requirement is flat over the whole frequency range that is of interest for the measurement. It is shown as a function of 


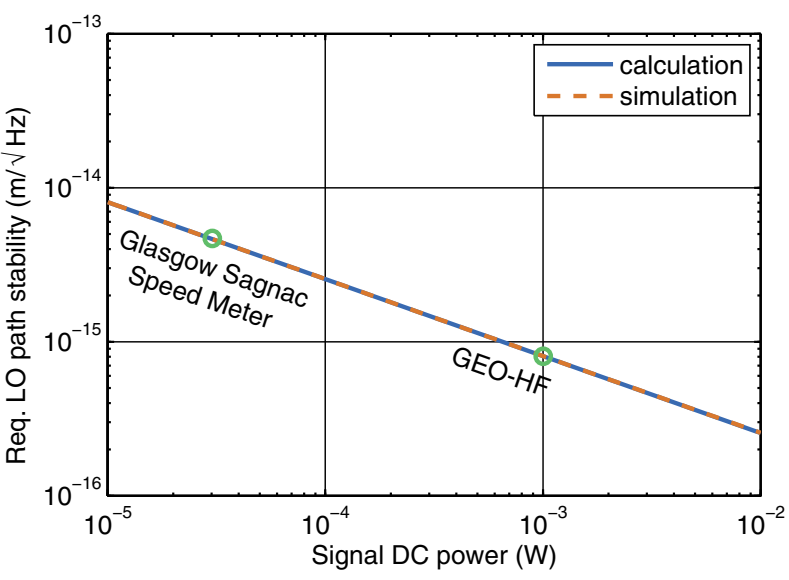

FIG. 2 (color online). Requirement on local-oscillator pathlength stability depending on the residual DC power level in the signal field. The blue curve is calculated from (17) for a wavelength of $1064 \mathrm{~nm}$, while the orange dashed curve is a simulated curve using the interferometry simulation tool Finesse [23]. We have indicated the requirements for the Glasgow Sagnac Speed Meter; see Sec. V below. In addition, we have added an indicative value for the high-frequency upgrade to GEO 600, based on based on the residual fundamental mode content in its output port [24].

$P_{\text {sig }}$ in Fig. 2, together with the results of a Finesse [23] simulation for this setup.

To verify this, we set up a small BHD experiment in which additional phase noise was injected into the local-oscillator path. The local-oscillator power was set to $0.55 \mathrm{~mW}$ (equivalent to $4.12 \mathrm{~V}_{\mathrm{DC}}$ on each photodiode). We injected a small amount of laser light into the signal beam, measuring less than $3 \mu \mathrm{W}$ or about a factor of 180 below the LO power level. The beat signal between LO and signal fields was used as an error signal to stabilize the BHD readout to the phase quadrature via a piezo-electric actuator (PZT) -mounted

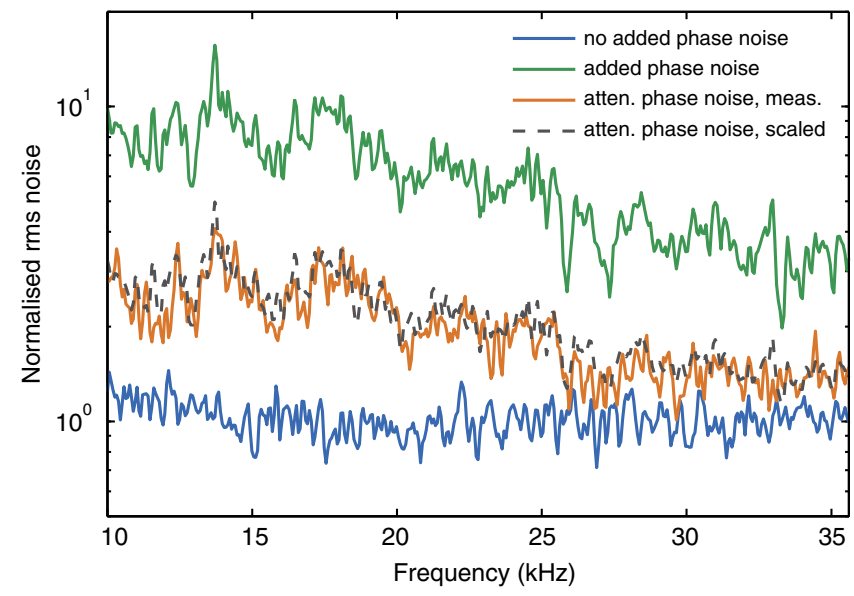

FIG. 3 (color online). Experimental demonstration of the effect of phase noise on the local-oscillator beam, and its dependence on signal beam power. The traces were measured with a FFT analyzer as $V_{\text {rms }}$, averaged 10 times and then normalized to the shot-noise level. mirror in the LO path. The resulting output noise spectrum was recorded with a spectrum analyzer. Figure 3 shows the spectrum (in blue) obtained for no additional phase noise, normalized such that the shot-noise level is at 1 . In the next step, white noise with an amplitude of $100 \mathrm{mV}_{p p}$ was added into the PZT drive voltage, giving the green trace. The trace's slope can be attributed to the declining transfer function of the PZT and its high-voltage amplifier. The excess phase noise on the LO is clearly visible. After further attenuating the signal beam with a neutral density filter with transmission $T \approx 10 \%$, the excess phase noise is significantly reduced (orange trace), which demonstrates that it scales with the signal power. Indeed, scaling the green curve with $\sqrt{T}$ (and taking the shot-noise contribution into account) results in the dashed grey curve, which closely fits the orange curve.

\section{BALANCED HOMODYNE READOUT IN ADVANCED LIGO}

As we have seen in Sec. I, balanced homodyne detection is a vital prerequisite for many quantum-noise reduction techniques. In addition, balanced homodyne readout offers the possibility to reduce technical noise limitations, such as photodiode detection noise and scattered light as we will discuss in the following paragraph.

In a foreseen upgrade to the Advanced LIGO gravitational wave detectors, frequency-dependent squeezed light will be used to improve the detectors' quantum-noise limited sensitivity [25]. The optimal balanced homodyne readout quadrature will then depend on both static and dynamic parameters of the interferometer, such as laser power, squeezing angle and filter cavity line width [26]. Indeed, the quadrature will also depend quite strongly on the chosen detection scenario which defines the optimal sensitivity; see, e.g., [27] for a discussion. As balanced homodyne detection provides the ability to finely tune the detected output quadrature, it would be the ideal readout tool in future detector upgrades and optimizations.

In addition, as Fritschel et al. [18] have pointed out, it will be increasingly difficult to maintain the sufficient noise margin between the photodiode electronic noise and the shot noise for high levels of squeezing. This margin is directly related to the $\mathrm{DC}$ voltage level after the photodiode transimpedance amplifier. In the presence of squeezing levels of about $10 \mathrm{~dB}$, challenging voltage levels exceeding $50 \mathrm{~V}_{\mathrm{DC}}$ will be encountered. Balanced homodyne detection would help in that the large DC offset can be directly subtracted before reaching the transimpedance amplifier stage [28]. Further technical benefits of balanced homodyne readout are connected to the reduction of light in the output port, because the dark-fringe offset that is necessary for DC readout can be eliminated. This would help mitigating scattered light noise which originates from the output optics [29], as well as reducing first-order coupling of beampointing noise on differential-wavefront sensors [30]. 
The DC signal power in Michelson-type interferometers such as Advanced LIGO is the result of two different processes; see Fig. 4. First, the light recombining at the main beam splitter can be offset in phase by slightly detuning the length of one of the interferometer arms. This dark-fringe offset leads to light in the gravitational wave signal's quadrature. Second, the recombining light can have a mismatch in amplitude, leading to some leakage light in the output field which is in the orthogonal quadrature to the gravitational wave signal. In total, this leads to a DC signal field in the output port that is oriented in some mixed quadrature orientation and both amplitudeand phase-quadrature noise couplings as discussed above have to be considered.

In Advanced LIGO, a local oscillator could be obtained from one of the beams that are reflected at the main beamsplitter antireflective coating [18]. The interferometer main laser is already amplitude stabilized to a very high degree [31], in addition to being frequency stabilized and filtered by the $4 \mathrm{~km}$-long power-recycling cavity. Thus, it should not be a problem to satisfy Eq. (12) for any remaining DC signal power that can reasonably be expected to occur in the interferometer output port.

The path-length stability from Eq. (17) sets a requirement on the suspension and control of any auxiliary optic

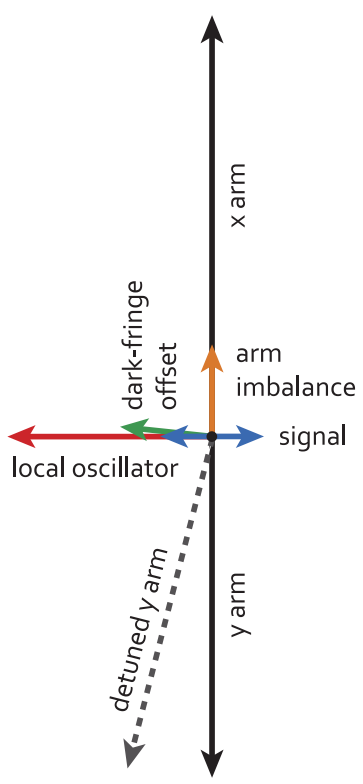

FIG. 4 (color online). Illustration of fields in the output port of a Michelson interferometer after recombination of the fields in the $x$ arm and $y$ arm. The externally supplied local oscillator (red) is chosen to be aligned to the gravitational wave signal's quadrature (blue). A slight detuning of one of the arms leads to a dark-fringe offset (green), corresponding to additional light in the signal quadrature which will amplify the LO's amplitude fluctuations. In contrast, an imbalance of the arms will result in additional light in the orthogonal quadrature (orange), and thus amplifies phase fluctuations of the local oscillator. that is not common to both local oscillator and signal path. Assuming that the signal DC power can be kept below $1 \mathrm{~mW}$, a displacement stability of about $1 \times 10^{-15} \mathrm{~m} / \sqrt{\mathrm{Hz}}$ would need to be achieved. This is beyond the capabilities of simple auxiliary suspensions, but within the requirements for, e.g., the input mode-cleaner suspensions [32].

\section{BALANCED HOMODYNE READOUT IN THE GLASGOW SAGNAC SPEED METER}

The Glasgow Sagnac Speed Meter Experiment [10] is a small-scale experiment with the goal of showing quantum backaction noise suppression in the Sagnac configuration [9], i.e., to demonstrate the feasibility of the speed meter concept for gravitational wave detectors. To achieve this goal, the experiment must be strongly dominated by radiation pressure forces at the frequencies of interest. This will be achieved by using light mirrors $(\approx 1 \mathrm{~g})$, suspended from multistage pendulums, in combination with high laser powers (several kilowatts inside highfinesse cavities).

For an ideal Sagnac interferometer, the output port stays at a dark fringe independent of any difference in the lengths of the two arms. Therefore, the Sagnac interferometer is unsuitable for the DC readout scheme used in Michelson interferometers, where a small arm-length difference is introduced to generate the required local oscillator [33]. Instead, the Sagnac interferometer is an obvious candidate for balanced homodyne readout with an externally supplied local oscillator.

Since every optic inside a Sagnac interferometer is seen by both the clockwise and counterclockwise beam, any loss in the two interferometer arms will equally appear in both propagation directions. Therefore, a loss mismatch between, e.g., the arm cavities will not lead to light in the output port-it will, however, affect the Sagnac speed meter's quantum-noise limited sensitivity [34]. Instead, and contrary to the Michelson interferometer, an asymmetry in the splitting ratio of the main 50:50 beam splitter will lead to light coupling into the output port, see Fig. 5: one propagation direction undergoes two reflections at this beam splitter, while the other undergoes two transmissions. For a mismatch in power reflectivity $\delta=R-0.5$, the resulting power at the Sagnac output scales as $4 P_{\text {in }} \delta^{2}$. Another coupling mechanism exists in the polarizing Sagnac interferometer topology, where insufficient separation between the two polarizations causes light to appear in the output port, as shown in [33].

In Fig. 6, we show RIN requirements for the localoscillator beam in the Glasgow Sagnac experiment, for different levels of beam-splitter asymmetry. Using the design parameters of this experiment, the expected power in the output port is $P_{\text {sig }} \approx 5.2 \mathrm{~W} \times \delta^{2}$. Since the readout quadrature is set to the signal quadrature (to within $\pm 2^{\circ}$ ), the requirements at high frequencies are relaxed by about 1 order of magnitude compared to Eq. (12), which gives the 


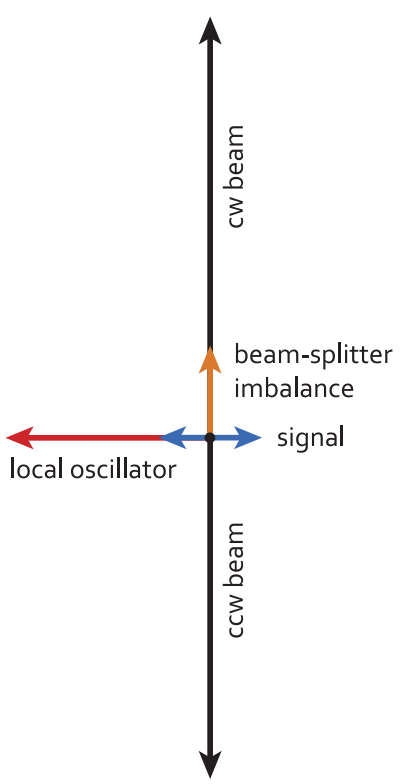

FIG. 5 (color online). Fields in the output port of a Sagnac interferometer, where the clockwise (cw) and counterclockwise (ccw) beams interfere destructively. The signal quadrature (blue) is readout with the help of an externally supplied local oscillator (red). An imbalance in the splitting ratio of the main beam splitter leads to light power in the orthogonal quadrature (orange). Contrary to the Michelson interferometer, there cannot be a static detuning of only one one of the two beams and so there is no DC field in the signal quadrature.

requirements for a readout of the orthogonal quadrature. Towards lower frequencies, the overall quantum noise in the output port rises strongly [34] and so a higher level of local-oscillator noise can be tolerated before it becomes dominant. We can therefore conclude that for the Glasgow Sagnac Speed Meter, the local oscillator does not have to

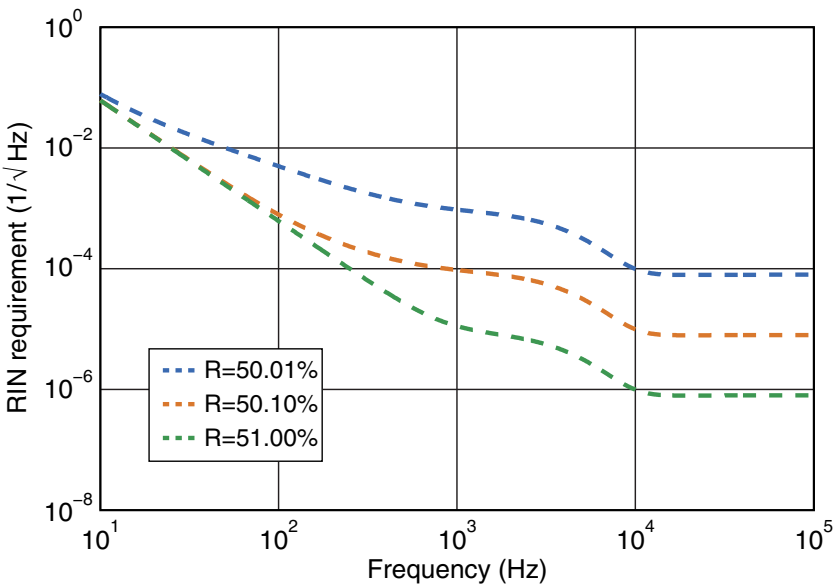

FIG. 6 (color online). Simulated requirements on the localoscillator amplitude stability for the Glasgow Sagnac Speed Meter experiment, depending on main beam-splitter imbalance. The traces were simulated with Finesse [23] and do not include noise sources other than quantum noise for the sensitivity modelling. be amplitude stabilized beyond what is needed for the main laser.

In terms of path stability, we assume that a beamsplitting imbalance of $\pm 0.2 \%$ is achievable. This would result in about $20 \mu \mathrm{W}$ DC signal power. According to Eq. (17) this leads to a displacement noise requirement of $<5.8 \times 10^{-14} \mathrm{~m} / \sqrt{\mathrm{Hz}}$ in the detection band from 100 to $1000 \mathrm{~Hz}$. We believe this is within the capabilities of our auxiliary beam steering suspensions, which we designed to achieve a horizontal displacement noise of about $1 \times 10^{-15} \mathrm{~m} / \sqrt{\mathrm{Hz}}$ at $100 \mathrm{~Hz}$.

\section{SUMMARY}

Novel aspects of quantum-noise reduction techniques in advanced gravitational wave detectors rely on a robust method for detecting arbitrary quadratures of the interferometer output field. Here we have investigated the challenges that arise when balanced homodyne readout is introduced to these detectors. In particular, we derived requirements for the amplitude noise and path-length stability of the local-oscillator field. We found that residual power in the signal path, caused by a contrast defect of the main interferometer, is the main driver of these requirements. We verified this result in a tabletop experiment, where we introduced additional phase noise in the localoscillator path. Within the examples of Advanced LIGO and the Glasgow Sagnac Speed Meter we showed that the derived requirements are manageable with current technology and do not exclude the application of balanced homodyne readout in gravitational wave detectors.

\section{ACKNOWLEDGMENTS}

The authors are grateful to Hartmut Grote for very valuable comments on this manuscript. The work described in this article is funded by the European Research Council (ERC-2012-StG: 307245). S.S. was funded by the Alexander von Humboldt Society and the International Max Planck Partnership (IMPP). We are grateful for support from the Science and Technology Facilities Council (Grant No. ST/L000946/1) and the ASPERA ET-R\&D project.

\section{APPENDIX A: DETAILED CALCULATION OF BALANCED HOMODYNE DETECTION}

Let us denote the two fields impinging onto the 50:50 beam splitter with $\boldsymbol{a}$ and $\boldsymbol{b}$. The relative phase between the two fields shall be $\phi$, which we write as an explicit phase $\boldsymbol{a} \rightarrow \boldsymbol{a} \mathrm{e}^{\mathrm{i} \phi}$. The two output fields $\boldsymbol{c}$ and $\boldsymbol{d}$ are then given by

$$
\begin{gathered}
\boldsymbol{c}=\frac{1}{\sqrt{2}}\left(\boldsymbol{a} \mathrm{e}^{\mathrm{i} \phi}+\boldsymbol{b}\right) \\
\boldsymbol{d}=\frac{1}{\sqrt{2}}\left(-\boldsymbol{a} \mathrm{e}^{\mathrm{i} \phi}+\boldsymbol{b}\right) .
\end{gathered}
$$


The light power in each output field is proportional to the number operators $\boldsymbol{c}^{\dagger} \boldsymbol{c}$ and $\boldsymbol{d}^{\dagger} \boldsymbol{d}$, respectively. These are given by

$$
\begin{aligned}
\boldsymbol{c}^{\dagger} \boldsymbol{c} & =\frac{1}{2}\left[\left(\boldsymbol{a}^{\dagger} \mathrm{e}^{-\mathrm{i} \phi}+\boldsymbol{b}^{\dagger}\right)\left(\boldsymbol{a} \mathrm{e}^{\mathrm{i} \phi}+\boldsymbol{b}\right)\right] \\
& =\frac{1}{2}\left[\boldsymbol{a}^{\dagger} \boldsymbol{a}+\boldsymbol{b}^{\dagger} \boldsymbol{b}+\boldsymbol{a}^{\dagger} \boldsymbol{b} \mathrm{e}^{-\mathrm{i} \phi}+\boldsymbol{a} \boldsymbol{b}^{\dagger} \mathrm{e}^{\mathrm{i} \phi}\right]
\end{aligned}
$$

and

$$
\begin{aligned}
\boldsymbol{d}^{\dagger} \boldsymbol{d} & =\frac{1}{2}\left[\left(-\boldsymbol{a}^{\dagger} \mathrm{e}^{-\mathrm{i} \phi}+\boldsymbol{b}^{\dagger}\right)\left(-\boldsymbol{a} \mathrm{e}^{\mathrm{i} \phi}+\boldsymbol{b}\right)\right] \\
& =\frac{1}{2}\left[\boldsymbol{a}^{\dagger} \boldsymbol{a}+\boldsymbol{b}^{\dagger} \boldsymbol{b}-\boldsymbol{a}^{\dagger} \boldsymbol{b} \mathrm{e}^{-\mathrm{i} \phi}-\boldsymbol{a} \boldsymbol{b}^{\dagger} \mathrm{e}^{\mathrm{i} \phi}\right] .
\end{aligned}
$$

We now decompose the operators into a classical amplitude and the quantum-mechanical fluctuations, $\boldsymbol{a}=\alpha+\delta \boldsymbol{a}$. Furthermore, we linearize the equations such that terms that are quadratic in the noise vanish, $\delta \boldsymbol{a} \delta \boldsymbol{b} \rightarrow 0$. With this, Eq. (A4) becomes

$$
\begin{aligned}
\boldsymbol{c}^{\dagger} \boldsymbol{c}= & \frac{1}{2}\left[\alpha^{2}+\alpha(\underbrace{\delta \boldsymbol{a}+\delta \boldsymbol{a}^{\dagger}}_{2 \delta \boldsymbol{X}_{1}^{a}}+\underbrace{\delta \boldsymbol{b} \mathrm{e}^{-\mathrm{i} \phi}+\delta \boldsymbol{b}^{\dagger} \mathrm{e}^{\mathrm{i} \phi}}_{2 \delta \boldsymbol{X}_{-\phi}^{b}})\right. \\
& +\beta^{2}+\beta(\underbrace{\delta \boldsymbol{b}+\delta \boldsymbol{b}^{\dagger}}_{2 \delta \boldsymbol{X}_{1}^{b}}+\underbrace{\delta \boldsymbol{a} \mathrm{e}^{\mathrm{i} \phi}+\delta \boldsymbol{a}^{\dagger} \mathrm{e}^{-\mathrm{i} \phi}}_{2 \delta \boldsymbol{X}_{\phi}^{a}}) \\
& +\underbrace{\alpha \beta \mathrm{e}^{\mathrm{i} \phi}+\alpha \beta \mathrm{e}^{-\mathrm{i} \phi}}_{2 \alpha \beta \cos \phi}] \\
= & \frac{1}{2}\left[\alpha^{2}+\beta^{2}+2 \alpha \beta \cos \phi+2 \alpha \delta \boldsymbol{X}_{1}^{a}+2 \beta \delta \boldsymbol{X}_{1}^{b}\right.
\end{aligned}
$$

$$
\left.+2 \alpha \delta \boldsymbol{X}_{-\phi}^{b}+2 \beta \delta \boldsymbol{X}_{\phi}^{a}\right]
$$

Similarly, Eq. (A6) becomes

$$
\begin{aligned}
\boldsymbol{d}^{\dagger} \boldsymbol{d}= & \frac{1}{2}\left[\alpha^{2}+\beta^{2}-2 \alpha \beta \cos \phi+2 \alpha \delta \boldsymbol{X}_{1}^{a}\right. \\
& \left.+2 \beta \delta \boldsymbol{X}_{1}^{b}-2 \alpha \delta \boldsymbol{X}_{-\phi}^{b}-2 \beta \delta \boldsymbol{X}_{\phi}^{a}\right] .
\end{aligned}
$$

The difference in photocurrents is therefore proportional to

$$
\boldsymbol{i}_{-}=\boldsymbol{c}^{\dagger} \boldsymbol{c}-\boldsymbol{d}^{\dagger} \boldsymbol{d}=2 \alpha \beta \cos \phi+2 \alpha \delta \boldsymbol{X}_{-\phi}^{b}+2 \beta \delta \boldsymbol{X}_{\phi}^{a}
$$

with a noise variance of

$$
\Delta^{2} \boldsymbol{i}_{-}=4 \alpha^{2} \Delta^{2} \delta \boldsymbol{X}_{-\phi}^{b}+4 \beta^{2} \Delta^{2} \delta \boldsymbol{X}_{\phi}^{a}
$$

\section{APPENDIX B: IMBALANCED SUBTRACTION}

We can model the effects of a nonideal common-mode rejection ratio by an imperfect subtraction in Eq. (A14), i.e., by writing

$$
\begin{aligned}
\boldsymbol{i}_{-, \epsilon}= & \boldsymbol{c}^{\dagger} \boldsymbol{c}-(1-\epsilon) \boldsymbol{d}^{\dagger} \boldsymbol{d} \\
= & \text { const }+\epsilon\left(\alpha \delta \boldsymbol{X}_{1}^{a}+\beta \delta \boldsymbol{X}_{1}^{b}\right) \\
& +(2-\epsilon)\left(\alpha \delta \boldsymbol{X}_{-\phi}^{b}+\beta \delta \boldsymbol{X}_{\phi}^{a}\right) .
\end{aligned}
$$

Comparing this result to Eq. (A14), we see that an additional noise term proportional to the amplitude noise $\delta \boldsymbol{X}_{1}$ in the two beams is introduced. Since $\alpha \gg \beta$ and often also $\Delta^{2} \delta \boldsymbol{X}_{1}^{a} \gg \Delta^{2} \delta \boldsymbol{X}_{1}^{b}$, the contribution from the local oscillator $\boldsymbol{a}$ will be dominating.
[1] A. Einstein, Über Gravitationswellen (Sitzungsberichte der Königlich Preußischen Akademie der Wissenschaften, Berlin, 1918).

[2] J. Aasi, B. P. Abbott, R. Abbott, T. Abbott, M. R. Abernathy, K. Ackley, C. Adams, T. Adams, P. Addesso, R. X. Adhikari, V. Adya, C. Affeldt, N. Aggarwal, O. D. Aguiar et al. (LIGO Scientific Collaboration), Classical Quantum Gravity 32, 074001 (2015).

[3] J. Abadie, B. P. Abbott, R. Abbot, T. D. Abbott, M. Abernathy, C. Adams, R. Adhikari, C. Affeldt, B. Allen, G. S. Allen, E. Amador Ceron, D. Amariutei, R. S. Amin et al. (LIGO Scientific Collaboration), Nat. Phys. 7, 962 (2011).
[4] J. Aasi, J. Abadie, B. Abbott, R. Abbott, T. Abbott, M. Abernathy, C. Adams, T. Adams, P. Addesso, R. Adhikari et al. (LIGO Scientific Collaboration), Nat. Photonics 7, 613 (2013).

[5] H. Grote, K. Danzmann, K. L. Dooley, R. Schnabel, J. Slutsky, and H. Vahlbruch, Phys. Rev. Lett. 110, 181101 (2013).

[6] H. J. Kimble, Y. Levin, A. B. Matsko, K. S. Thorne, and S. P. Vyatchanin, Phys. Rev. D 65, 022002 (2001).

[7] V. B. Braginsky, Y. I. Vorontsov, and K. S. Thorne, Science 209, 547 (1980).

[8] V. B. Braginsky and F. J. Khalili, Phys. Lett. A 147, 251 (1990). [9] Y. Chen, Phys. Rev. D 67, 122004 (2003). 
[10] C. Gräf et al., Classical Quantum Gravity 31, 215009 (2014).

[11] S. Hild, H. Grote, J. Degallaix, S. Chelkowski, K. Danzmann, A. Freise, M. Hewitson, J. Hough, H. Lück, M. Prijatelj et al., Classical Quantum Gravity 26, 055012 (2009).

[12] T. T. Fricke, N. D. Smith-Lefebvre, R. Abbott, R. Adhikari, K. L. Dooley, M. Evans, P. Fritschel, V. V. Frolov, K. Kawabe, J.S. Kissel et al., Classical Quantum Gravity 29, 065005 (2012).

[13] See, e. g., U. Leonhardt, Measuring the Quantum State of Light (Cambridge University Press, Cambridge, 1997).

[14] J. DiGuglielmo, A. Samblowski, B. Hage, C. Pineda, J. Eisert, and R. Schnabel, Phys. Rev. Lett. 107, 240503 (2011).

[15] H.-A. Bachor and T. C. Ralph, A Guide to Experiments in Quantum Optics, 2nd ed. (Wiley-VCH, New York, 2004).

[16] S. Ast, M. Mehmet, and R. Schnabel, Opt. Express 21, 13572 (2013).

[17] M. Stefszky, C. Mow-Lowry, S. Chua, D. Shaddock, B. Buchler, H. Vahlbruch, A. Khalaidovski, R. Schnabel, P. Lam, and D. McClelland, Classical Quantum Gravity 29, 145015 (2012).

[18] P. Fritschel, M. Evans, and V. Frolov, Opt. Express 22, 4224 (2014).

[19] C. Gerry and P. Knight, Introductory Quantum Optics (Cambridge University Press, Cambridge, 2005).

[20] P. Kwee, B. Willke, and K. Danzmann, Opt. Lett. 34, 2912 (2009).

[21] K. McKenzie, M. B. Gray, P. K. Lam, and D. E. McClelland, Appl. Opt. 46, 3389 (2007).

[22] S. L. Danilishin and F. Y. Khalili, Living Rev. Relativity 15, 5 (2012).
[23] A. Freise, G. Heinzel, H. Lück, R. Schilling, B. Willke, and K. Danzmann, Classical Quantum Gravity 21, S1067 (2004); the program is available at http://www.gwoptics .org/finesse.

[24] H. Grote (personal communication).

[25] LIGO Scientific Collaboration, Report No. LIGO-T1400316, 2014.

[26] N. Voronchev, S. Danilishin, and F. Khalili, Moscow Univ. Phys. Bull. 69, 519 (2014).

[27] N. V. Voronchev, S. P. Tarabrin, and S. L. Danilishin, arXiv: 1503.01062.

[28] Alternative investigations are under way on how to reduce the DC current in the transimpedance stage of photo diode electronics; see D. McClelland, Report No. LIGOG1500371, 2015.

[29] D. Martynov, Report No. LIGO-G1500656, 2015.

[30] H. Grote and the LIGO Scientific Collaboration, Classical Quantum Gravity 27, 084003 (2010).

[31] P. Kwee, C. Bogan, K. Danzmann, M. Frede, H. Kim, P. King, J. Pöld, O. Puncken, R. L. Savage, F. Seifert, P. Wessels, L. Winkelmann, and B. Willke, Opt. Express 20, 10617 (2012).

[32] P. Fritschel and D. Coyne, Report No. LIGO-T010075-v3, 2015.

[33] There are, however, some proposals on how to produce a local oscillator in the signal quadrature; see M. Wang, C. Bond, D. Brown, F. Brückner, L. Carbone, R. Palmer, and A. Freise, Phys. Rev. D 87, 096008 (2013).

[34] S. L. Danilishin, C. Gräf, S. S. Leavey, J. Hennig, E. A. Houston, D. Pascucci, S. Steinlechner, J. Wright, and S. Hild, New J. Phys. 17, 043031 (2015). 